\title{
Impact of Localizing General Medical Teams to a Single Nursing Unit
}

\author{
Siddhartha Singh, MD, MS ${ }^{1,2,4 x}$, Sergey Tarima, PhD'1, Vipulkumar Rana, MD4, David S. Marks, MD, MBA4,

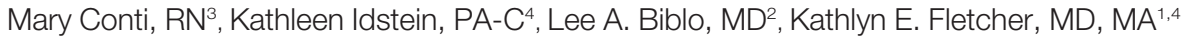

${ }^{1}$ Center for Patient Care and Outcomes Research, Medical College of Wisconsin, Milwaukee, Wisconsin; ${ }^{2}$ Department of Clinical Affairs, Medical College Physicians, Medical College of Wisconsin, Milwaukee, Wisconsin; ${ }^{3} J$ oint Clinical Quality Office, Froedtert Hospital, Milwaukee, Wisconsin; ${ }^{4}$ Department of Medicine, Medical College of Wisconsin, Milwaukee, Wisconsin.

BACKGROUND: Localization of general medical inpatient teams is an attractive way to improve inpatient care but has not been adequately studied.

OBJECTIVE: To evaluate the impact of localizing general medical teams to a single nursing unit.

DESIGN: Quasi-experimental study using historical and concurrent controls.

SETTING: A 490-bed academic medical center in the midwestern United States.

PATIENTS: Adult, general medical patients, other than those with sickle cell disease, admitted to medical teams staffed by a hospitalist and a physician assistant (PA).

INTERVENTION: Localization of patients assigned to 2 teams to a single nursing unit.

MEASUREMENTS: Length of stay (LOS), 30-day risk of readmission, charges, pages to teams, encounters, relative value units (RVUs), and steps walked by PAs.
RESULTS: Localized teams had 0.89 (95\% confidence interval [Cl], 0.37-1.41) more patient encounters and generated 2.20 more RVUs per day (CI, 1.10-3.29) compared to historical controls; and 1.02 ( $\mathrm{Cl}, 0.46-1.58)$ more patient encounters and generated 1.36 more RVUs per day $(\mathrm{Cl}, 0.17-2.55)$ compared to concurrent controls. Localized teams received $51 \%(\mathrm{Cl}, 48-54)$ fewer pages during the workday. LOS may have been approximately $10 \%$ higher for localized teams. Risk of readmission within 30 days and charges incurred were no different. PAs possibly walked fewer steps while localized.

CONCLUSION: Localization of medical teams led to higher productivity and better workflow, but did not significantly impact readmissions or charges. It may have had an unintended negative impact on hospital efficiency; this finding deserves further study. Journal of Hospital Medicine 2012;7:551-556. (C) 2012 Society of Hospital Medicine
Localizing inpatient general medical teams to nursing units has high intuitive validity for improving physician productivity, hospital efficiency, and patient outcomes. Motion or the moving of personnel between tasks-so prominent if teams are not localized-is 1 of the 7 wastes in "lean thinking." In a time-motion study, where hospitalists cared for patients on up to 5 different wards, O'Leary et $\mathrm{al}^{2}$ have reported large parts of hospitalists' workdays spent in indirect patient care $(69 \%)$, paging $(13 \%)$, and travel $(3 \%)$. Localization could increase the amount of time available for direct patient care, decrease time spent for (and interruptions due to) paging, and decrease travel time, all leading to greater productivity.

O'Leary et $\mathrm{al}^{3}$ have also reported the beneficial effects of localization of medical inpatients on communication between nurses and physicians, who could

*Address for correspondence and reprint requests: Siddhartha Singh, MD, MS, Medical College Physicians, The Medical College of Wisconsin, 9200 West Wisconsin Ave, Milwaukee, WI 53226; Telephone: 414-805-0844; Fax: 414-805-0454; E-mail: ssingh@mcw.edu Additional Supporting Information may be found in the online version of this article.

Received: January 18, 2012; Revised: March 8, 2012; Accepted: April 13, 2012

2012 Society of Hospital Medicine DOI 10.1002/jhm.1948

Published online in Wiley Online Library (Wileyonlinelibrary.com). identify each other more often, and reported greater communication (specifically face-to-face communication) with each other following localization. This improvement in communication and effective multidisciplinary rounds could lead to safer care ${ }^{4}$ and better outcomes.

Further investigations about the effect of localization are limited. Roy et $\mathrm{al}^{5}$ have compared the outcomes of patients localized to 2 inpatient "pods" medically staffed by hospitalists and physician assistants (PAs) to geographically dispersed, but structurally different, house staff teams. They noticed significantly lower costs, slight but nonsignificant increase in length of stay, and no difference in mortality or readmissions, but it is impossible to tease out the affect of localization versus the affect of team composition. In a before-andafter study, Findlay et $\mathrm{al}^{6}$ have reported a decrease in mortality and complication rates in clinically homogenous surgical patients (proximal hip fractures) when cared for by junior trainee physicians localized to a unit, but their experience cannot be extrapolated to the much more diverse general medical population.

In our hospital, each general medical team could admit patients dispersed over 14 different units. An internal group, commissioned to evaluate our hospitalist practice, recommended reducing this dispersal to improve physician productivity, hospital efficiency, 
and outcomes of care. We therefore conducted a project to evaluate the impact of localizing general medical inpatient teams to a single nursing unit.

\section{METHODS}

\section{Setting}

We conducted our project at a 490 bed, urban academic medical center in the midwestern United States where of the 10 total general medical teams, 6 were traditional resident-based teams and 4 consisted of a hospitalist paired with a PA (H-PA teams). We focused our study on the 4 $\mathrm{H}-\mathrm{PA}$ teams. The hospitalists could be assigned to any $\mathrm{H}-$ PA team and staffed them for 2 weeks (including weekends). The PAs were always assigned to the same team but took weekends off. An in-house hospitalist provided overnight cross-coverage for the H-PA teams. Prior to our intervention, these teams could admit patients to any of the 14 nursing units at our hospital. They admitted patients from 7 AM to $3 \mathrm{PM}$, and also accepted care of patients admitted overnight after the resident teams had reached their admission limits (overflow). A Faculty Admitting Medical Officer (AMO) balanced the existing workload of the teams against the number and complexity of incoming patients to decide team assignment for the patients. The AMO was given guidelines ("soft" caps) to limit total admissions to H-PA teams to 5 per team per day ( 3 on a weekend), and to not exceed a total patient census of 16 for an H-PA team.

\section{Intervention}

Starting April 1, 2010, until July 15, 2010, we localized patients admitted to 2 of our 4 H-PA teams on a single 32-bed nursing unit. The patients of the other 2 H-PA teams remained dispersed throughout the hospital.

\section{Transition}

April 1, 2010 was a scheduled switch day for the hospitalists on the H-PA teams. We took advantage of this switch day and reassigned all patients cared for by H-PA teams on our localized unit to the 2 localized teams. Similarly, all patients on nonlocalized units cared for by H-PA teams were reassigned to the 2 nonlocalized teams. All patients cared for by resident teams on the localized unit, that were anticipated to be discharged soon, stayed until discharge; those that had a longer stay anticipated were transferred to a nonlocalized unit.

\section{Patient Assignment}

The 4 H-PA teams continued to accept patients between $7 \mathrm{AM}$ and $3 \mathrm{PM}$, as well as overflow patients. Patients with sickle cell crises were admitted exclusively to the nonlocalized teams, as they were cared for on a specialized nursing unit. No other patient characteristic was used to decide team assignment.

The AMO balanced the existing workload of the teams against the number and complexity of incoming patients to decide team assignment for the patients, but if these factors were equivocal, the AMO was now asked to preferentially admit to the localized teams. The admission "soft" cap for the H-PA teams remained the same (5 on weekdays and 3 on weekends). The "soft" cap on the total census of 16 patients for the nonlocalized teams remained, but we imposed "hard" caps on the total census for the localized teams. These "hard" caps were 16 for each localized team for the month of April (to fill a 32-bed unit), then decreased to 12 for the month of May, as informal feedback from the teams suggested a need to decrease workload, and then rebalanced to 14 for the remaining study period.

\section{Evaluation Clinical Outcomes}

Using both concurrent and historical controls, we evaluated the impact of localization on the following clinical outcome measures: length of stay (LOS), charges, and 30-day readmission rates.

Inclusion Criteria. We included all patients assigned to localized and nonlocalized teams between the period April 1, 2010 to July 15, 2010, and discharged before July 16, 2010, in our intervention group and concurrent control group, respectively. We included all patients assigned to any of the $4 \mathrm{H}-\mathrm{PA}$ teams during the period January 1, 2010 and March 31, 2010 in the historical control group.

Exclusion Criteria. From the historical control group, we excluded patients assigned to one particular H-PA team during the period January 1, 2010 to February 28, 2010, during which the PA assigned to that team was on leave. We excluded, from all groups, patients with a diagnosis of sickle cell disease and hospitalizations that straddled the start of the intervention. Further, we excluded repeat admissions for each patient.

Data Collection. We used admission logs to determine team assignment and linked them to our hospital's discharge abstract database to get patient level data. We grouped the principal diagnosis, International Classification of Diseases, Ninth Revision, Clinical Modification (ICD-9-CM) codes into clinically relevant categories using the "Healthcare Cost and Utilization Project Clinical Classification Software for ICD-9-CM (Rockville, MD, www.hcup-us.ahrq.gov/ toolssoftware/ccs/ccs.jsp)." We created comorbidity measures using "Healthcare Cost and Utilization Project Comorbidity Software, version 3.4 (Rockville, MD, www.hcup-us.ahrq.gov/toolssoftware/comorbidity/comorbidity.jsp)."

We calculated LOS by subtracting the discharge day and time from the admission day and time. We summed all charges accrued during the entire hospital stay, but did not include professional fees. The LOS and charges included time spent and charges accrued 
TABLE 1. Characteristics of Patients Admitted to Localized Teams and Control Groups

\begin{tabular}{|c|c|c|c|c|}
\hline & $\begin{array}{l}\text { Historical } \\
\text { Control }\end{array}$ & $\begin{array}{l}\text { Intervention } \\
\text { Localized } \\
\text { Teams }\end{array}$ & $\begin{array}{l}\text { Concurrent } \\
\text { Control }\end{array}$ & $P$ Value \\
\hline Patients & 783 & 565 & 478 & - \\
\hline \multicolumn{5}{|l|}{ Age groups, $n(\%)$} \\
\hline$<30$ & $65(8.3)$ & $37(6.6)$ & $46(9.6)$ & \\
\hline $30-39$ & $76(9.7)$ & $62(11.0)$ & $47(9.8)$ & \\
\hline $60-69$ & $119(15.2)$ & $84(14.9)$ & $76(16.0)$ & \\
\hline $70-79$ & $100(12.8)$ & $62(11.0)$ & $58(12.1)$ & \\
\hline $80-89$ & $113(14.4)$ & $95(16.8)$ & $51(10.7)$ & \\
\hline$>89$ & $34(4.3)$ & $16(2.88)$ & $14(2.9)$ & \\
\hline Female gender, $n(\%)$ & $434(55.4)$ & $327(57.9)$ & $264(55.2)$ & 0.602 \\
\hline Race: Black, n (\%) & $285(36.4)$ & $229(40.5)$ & $200(41.8)$ & 0.111 \\
\hline Medicaid & $179(22.8)$ & $126(22.3)$ & $117(24.5)$ & \\
\hline Uninsured & $54(7.3)$ & $60(10.6)$ & $42(8.8)$ & \\
\hline Weekend admission, $n(\%)$ & $137(17.5)$ & $116(20.5)$ & $65(13.6)$ & 0.013 \\
\hline Weekend discharge, $n(\%)$ & $132(16.9)$ & $107(18.9)$ & $91(19.0)$ & 0.505 \\
\hline \multicolumn{5}{|l|}{ Source of admission } \\
\hline ED, $n(\%)$ & $654(83.5)$ & $450(79.7)$ & $370(77.4)$ & 0.022 \\
\hline No ICU stay, n (\%) & $600(76.6)$ & $440(77.9)$ & $383(80.1)$ & 0.348 \\
\hline \multicolumn{5}{|l|}{ Admission time, $n(\%)$} \\
\hline 0000-0559 & $239(30.5)$ & $208(36.8)$ & $172(36.0)$ & \\
\hline $0600-1159$ & $296(37.8)$ & $157(27.8)$ & $154(32.2)$ & 0.007 \\
\hline $1200-1759$ & $183(23.4)$ & $147(26.0)$ & $105(22.0)$ & \\
\hline $1800-2359$ & $65(8.3)$ & $53(9.4)$ & $47(9.8)$ & \\
\hline \multicolumn{5}{|l|}{ Discharge time, $\mathrm{n}(\%)$} \\
\hline 4 & Pneumonia (2.8) & Diabetes w/cm (4.1) & Pneumonia (3.3) & \\
\hline 5 & UTI (2.7) & COPD (3.2) & UTI (2.9) & \\
\hline
\end{tabular}

Abbreviations: COPD, chronic obstructive pulmonary disease; ED, emergency department; ICU, intensive care unit; IQR, interquartile range; $n$, number; $n / a$, not applicable; UTI, urinary tract infection; w/cm, with complications.

in the intensive care unit (ICU). As ICU care was not under the control of the general medical teams and could have a significant impact on outcomes reflecting resource utilization, we compared LOS and charges only for 2 subsets of patients: patients not initially admitted to ICU before care by medical teams, and patients never requiring ICU care. We considered any repeat hospitalization to our hospital within 30 days following a discharge to be a readmission, except those for a planned procedure or for inpatient rehabilitation. We compared readmission rates for all patients irrespective of ICU stay, as discharge planning for all patients was under the direct control of the general medical teams.

Data Analysis. We performed unadjusted descriptive statistics using medians and interquartile ranges for continuous variables, and frequencies and percentages for categorical variables. We used chi-square tests of association, and Kruskal-Wallis analysis of variance, to compare baseline characteristics of patients assigned to localized and control teams.

We used regression models with random effects to risk adjust for a wide variety of variables. We included age, gender, race, insurance, admission source, time, day of week, discharge time, and total number of comorbidities as fixed effects in all models. We then added individual comorbidity measures one by one as fixed effects, including them only if significant at $P<0.01$. We always added a variable identifying the admitting physician as a random effect, to account for dependence between admissions to the same physician. We log transformed LOS and charges because they were extremely skewed in nature. We analyzed readmissions after excluding patients who 


\begin{tabular}{|c|c|c|c|c|}
\hline & Historical Control & Intervention Localized Teams & Concurrent Control & $P$ Value \\
\hline 30-day readmissions n (\%) & $118(15.3)$ & $69(12.5)$ & $66(14.0)$ & 0.346 \\
\hline \multicolumn{5}{|l|}{ Charges: excluding patients initially admitted to ICU } \\
\hline Median (IQR) in \$ & $9346(6216-14,520)$ & $9724(6657-15,390)$ & $9902(6611-15,670)$ & 0.393 \\
\hline \multicolumn{5}{|l|}{ Charges: excluding all patients with an ICU stay } \\
\hline Median (IQR) in \$ & $9270(6187-13,990)$ & $9509(6601-14,940)$ & $9846(6580-15,400)$ & 0.283 \\
\hline \multicolumn{5}{|l|}{ Length of stay: excluding patients initially admitted to ICU } \\
\hline Median (IQR) in days & $1.81(1.22-3.35)$ & $2.16(1.21-4.02)$ & $1.89(1.19-3.50)$ & 0.214 \\
\hline \multicolumn{5}{|l|}{ Length of stay: excluding all patients with an ICU stay } \\
\hline Median (IQR) in days & $1.75(1.20-3.26)$ & $2.12(1.20-3.74)$ & $1.84(1.19-3.42)$ & 0.236 \\
\hline
\end{tabular}

Abbreviations: ICU, intensive care unit; IQR, interquartile range; $n$, number; \$, United States dollars.

died. We evaluated the affect of our intervention on clinical outcomes using both historical and concurrent controls. We report $P$ values for both overall 3-way comparisons, as well as each of the 2-way comparisons-intervention versus historical control and intervention versus concurrent control.

\section{Productivity and Workflow Measures}

We also evaluated the impact of localization on the following productivity and workflow measures: number of pages received, number of patient encounters, relative value units (RVUs) generated, and steps walked by PAs.

Data Collection. We queried our in-house paging systems for the number of pages received by intervention and concurrent control teams between $7 \mathrm{AM}$ and $6 \mathrm{PM}$ (usual workday). We queried our professional billing data to determine the number of encounters per day and RVUs generated by the intervention, as well as historical and concurrent control teams, as a measure of productivity.

During the last 15 days of our intervention (July 1July 15, 2010), we received 4 pedometers and we asked the PAs to record the number of steps taken during their workday. We chose PAs, rather than physicians, as the PAs had purely clinical duties and their walking activity would reflect activity for solely clinical purposes.

Data Analysis. For productivity and workflow measures, we adjusted for the day of the week and used random effects models to adjust for clustering of data by physician and physician assistant.

Statistical Software. We performed the statistical analysis using R software, versions 2.9.0 (The R Project for Statistical Computing, Vienna, Austria, http://www.Rproject.org).

Ethical Concerns. The study protocol was approved by our institutional review board.

\section{RESULTS}

\section{Study Population}

There were 2431 hospitalizations to the 4 H-PA teams during the study period. Data from 37 hospitalizations was excluded because of missing data. After applying all exclusion criteria, our final study sample consisted of a total of 1826 first hospitalizations for patients: 783 historical controls, 478 concurrent controls, and 565 localized patients.

Patients in the control groups and intervention group were similar in age, gender, race, and insurance status. Patients in the intervention group were more likely to be admitted over the weekend, but had similar probability of being discharged over the weekend or having had an ICU stay. Historical controls were admitted more often between $6 \mathrm{Am}$ and 12 noon, while during the intervention period, patients were more likely to be admitted between midnight and 6 AM. The discharge time was similar across all groups. The 5 most common diagnoses were similar across the groups (Table 1).

\section{Clinical Outcomes Unadjusted Analyses}

The risk of 30-day readmission was no different between the intervention and control groups. In patients without an initial ICU stay, and without any ICU stay, charges incurred and LOS were no different between the intervention and control groups (Table 2).

\section{Adjusted Analysis}

The risk of 30-day readmission was no different between the intervention and control groups. In patients without an initial ICU stay, and without any ICU stay, charges incurred were no different between the intervention and control groups; LOS was about $11 \%$ higher in the localized group as compared to historical controls, and about $9 \%$ higher as compared to the concurrent control group. The difference in LOS was not statistically significant on an overall 3-way comparison (Table 3).

\section{Productivity and Workflow Measures Unadjusted Analyses}

The localized teams received fewer pages as compared to concurrently nonlocalized teams. Localized teams had more patient encounters per day and generated more RVUs per day as compared to both historical and concurrent control groups. Physician assistants on localized teams took fewer steps during their work day (Table 4). 


\begin{tabular}{|c|c|c|c|}
\hline & \multicolumn{2}{|c|}{ Localized Teams in Comparison to } & \multirow[b]{2}{*}{ Overall $P$ Value } \\
\hline & Historical Control & Concurrent Control & \\
\hline 30-day risk of readmission OR (CI) & $0.85(0.61-1.19)$ & $0.94(0.65-1.37)$ & 0.630 \\
\hline Pvalue & 0.351 & 0.751 & \\
\hline \multicolumn{4}{|c|}{ Charges: excluding patients initially admitted to ICU } \\
\hline $\pm \%$ change & $2 \%$ higher & $4 \%$ lower & 0.367 \\
\hline (Cl) & ( $6 \%$ lower to $11 \%$ higher) & (12\% lower to 5\%higher) & \\
\hline Pvalue & 0.572 & 0.427 & \\
\hline \multicolumn{4}{|c|}{ Charges: excluding all patients with an ICU stay } \\
\hline $\pm \%$ change & $2 \%$ higher & $5 \%$ lower & 0.314 \\
\hline (Cl) & (6\% lower to $10 \%$ higher) & (13\% lower to 4\% higher) & \\
\hline Pvalue & 0.695 & 0.261 & \\
\hline \multicolumn{4}{|c|}{ Length of stay: excluding patients initially admitted to ICU } \\
\hline $\pm \%$ change & $11 \%$ higher & $9 \%$ higher & 0.105 \\
\hline (CI) & (1\% to 22\% higher) & (3\% lower to $21 \%$ higher) & \\
\hline Pvalue & 0.038 & 0.138 & \\
\hline \multicolumn{4}{|c|}{ Length of stay: excluding all patients with an ICU stay } \\
\hline $\pm \%$ change & $10 \%$ higher & $8 \%$ higher & 0.133 \\
\hline (Cl) & (0\% to 22\% higher) & (3\% lower to 20\% higher) & \\
\hline Pvalue & 0.047 & 0.171 & \\
\hline
\end{tabular}

Abbreviations: $\mathrm{Cl}$, confidence interval; ICU, intensive care unit; $\mathrm{OR}$, odds ratio.

\begin{tabular}{|c|c|c|c|c|}
\hline & Historical Control & Intervention Localized Teams & Concurrent Control & $P$ Value \\
\hline Pages received/day (7 AM-6 PM) Median (IQR) & No data & $15(9-21)$ & $28(12.5-40)$ & $<0.001$ \\
\hline Total encounters/day Median (IQR) & $10(8-13)$ & $12(10-13)$ & $11(9-13)$ & $<0.001$ \\
\hline \multicolumn{5}{|l|}{ RVU/day } \\
\hline Mean (SD) & $19.9(6.76)$ & $22.6(5.6)$ & $21.2(6.7)$ & $<0.001$ \\
\hline Steps/day Median (IOR) & No data & $4661(3922-5166)$ & $5554(5060-6544)$ & $<0.001$ \\
\hline
\end{tabular}

\section{Adjusted Analysis}

On adjusting for clustering by physician and day of week, the significant differences in pages received, total patient encounters, and RVUs generated persisted, while the difference in steps walked by PAs was attenuated to a statistically nonsignificant level (Table 5). The increase in RVU productivity was sustained through various periods of "hard caps" (data not shown).

\section{DISCUSSION}

We found that general medical patients admitted to $\mathrm{H}$ PA teams and localized to a single nursing unit had similar risk of 30-day readmission and charges, but may have had a higher length of stay compared to historical and concurrent controls. The localized teams received far fewer pages, had more patient encounters, generated more RVUs, and walked less during their work day. Taken together, these findings imply that in our study, localization led to greater team productivity and a possible decrease in hospital efficiency, with no significant impact on readmissions or charges incurred.

The higher productivity was likely mediated by the preferential assignments of more patients to the localized teams, and improvements in workflow (such as fewer pages and fewer steps walked), which allowed them to provide more care with the same resources as the control teams. Kocher and Sahni ${ }^{7}$ recently pointed out that the healthcare sector has experienced no gains in labor productivity in the past 20 years. Our intervention fits their prescription for redesigning healthcare delivery models to achieve higher productivity.

The possibility of a higher LOS associated with localization was a counterintuitive finding, and similar to that reported by Roy et al. ${ }^{5}$ We propose 3 hypotheses to explain this:

1. Selection bias: Higher workload of the localized teams led to compromised efficiency and a higher length of stay (eg, localized teams had fewer observation admissions, more hospitalizations with an ICU stay, and the AMO was asked to preferentially admit patients to localized teams).

2. Localization provided teams the opportunity to spend more time with their patients (by decreasing non-value-added tasks) and to consequently address more issues before transitioning to outpatient care, or to provide higher quality of care.

3. Gaming: By having a "hard cap" on total number of occupied beds, we provided a perverse incentive to the localized teams to retain patients longer to keep 
TABLE 5. Adjusted Comparisons of Productivity and Workflow Outcomes Between Localized Teams and Control Groups

\begin{tabular}{|c|c|c|c|}
\hline & \multicolumn{2}{|c|}{ Localized Teams in Comparison to } & \multirow[b]{2}{*}{$\begin{array}{l}\text { Overall } \\
P \text { Value }\end{array}$} \\
\hline & $\begin{array}{l}\text { Historical } \\
\text { Control }\end{array}$ & $\begin{array}{l}\text { Concurrent } \\
\text { Control }\end{array}$ & \\
\hline Pvalue & & $P<0.001$ & - \\
\hline Total encounters & 0.89 more & 1.02 more & \\
\hline $\pm N(C l)$ & $(0.37-1.41)$ & $(0.46-1.58)$ & \\
\hline Pvalue & $P<0.001$ & $P<0.001$ & $P<0.001$ \\
\hline $\pm N(C l)$ & $(1.10-3.29)$ & $(0.17-2.55)$ & \\
\hline Pvalue & $P<0.001$ & $P=0.024$ & $P<0.001$ \\
\hline Steps/day & & 1186 fewer (791 more to & \\
\hline $\pm N(C)$ & No data & 3164 fewer) & \\
\hline Pvalue & & $P=0.240$ & - \\
\hline
\end{tabular}

assigned beds occupied, thereby delaying new admissions to avoid higher workload.

Our study cannot tell us which of these hypotheses represents the dominant phenomenon that led to this surprising finding. Hypothesis 3 is most worrying, and we suggest that others looking to localize their medical teams consider the possibility of unintended perverse incentives.

Differences were more pronounced between the historical control group and the intervention group, as opposed to the intervention group and concurrent controls. This may have occurred if we contaminated the concurrent control by decreasing the number of units they had to go to, by sequestering 1 unit for the intervention team.

Our report has limitations. It is a nonrandomized, quasi-experimental investigation using a single institution's administrative databases. Our intervention was small in scale (localizing 2 out of 10 general medical teams on 1 out of 14 nursing units). What impact a wider implementation of localization may have on emergency department throughput and hospital occupancy remains to be studied. Nevertheless, our research is the first report, to our knowledge, investigating a wide variety of outcomes of localizing inpatient medical teams, and adds significantly to the lim- ited research on this topic. It also provides significant operational details for other institutions to use when localizing medical teams.

We conclude that our intervention of localization of medical teams to a single nursing unit led to higher productivity and better workflow, but did not impact readmissions or charges incurred. We caution others designing similar localization interventions to protect against possible perverse incentives for inefficient care.

Disclosure: Nothing to report.

\section{References}

1. Bush RW. Reducing waste in US health care systems. JAMA. 2007; 297(8):871-874.

2. O'Leary KJ, Liebovitz DM, Baker DW. How hospitalists spend their time: insights on efficiency and safety. J Hosp Med. 2006;1(2):88-93.

3. O'Leary K, Wayne D, Landler M, et al. Impact of localizing physicians to hospital units on nurse-physician communication and agreement on the plan of care. J Gen Intern Med. 2009;24(11):1223-1227.

4. O'Leary KJ, Buck R, Fligiel HM, et al. Structured interdisciplinary rounds in a medical teaching unit: improving patient safety. Arch Intern Med. 2011;171(7):678-684.

5. Roy CL, Liang CL, Lund M, et al. Implementation of a physician assistant/hospitalist service in an academic medical center: impact on efficiency and patient outcomes. J Hosp Med. 2008;3(5):361-368.

6. Findlay JM, Keogh MJ, Boulton C, Forward DP, Moran CG. Wardbased rather than team-based junior surgical doctors reduce mortality for patients with a fracture of the proximal femur: results from a twoyear observational study. J Bone Joint Surg Br. 2011;93-B(3):393-398.

7. Kocher R, Sahni NR. Rethinking health care labor. N Engl J Med. 2011;365(15):1370-1372. 